\title{
Linfangioma de cabeça e pescoço: levantamento de casos
}

\author{
Lymphangioma of the head and neck: survey of cases
}

\author{
Luiza Helena Rebelato Krakhecke* \\ João Paulo De Carli** \\ Bethânia Molin Giaretta De Carli** \\ Soluete Oliveira da Silva ${ }^{* * * *}$
}

\section{Resumo}

Os linfangiomas são tumores benignos de vasos linfáticos que afetam geralmente a cabeça e o pescoço. Esses podem ter origem congênita ou constituir lesões que se desenvolvem ao longo da vida, acometendo sobretudo crianças. Podem ser tratados cirurgicamente, por substâncias esclerosantes ou por meio de radioterapia, no entanto, sua recidiva acontece entre $10 \%$ e $40 \%$ dos casos. Materiais e método: o objetivo deste estudo foi realizar uma pesquisa epidemiológica dos casos de linfangioma registrados nos laboratórios de Patologia do Instituto de Ciências Biológicas da Universidade de Passo Fundo (ICB/UPF) e do Hospital São Vicente de Paulo de Passo Fundo/RS (HSVP) desde o ano 1987 até 2012. Resultados: ao todo, foram catalogados 78 casos de linfangioma. Desses, 69 acometeram a região de cabeça e de pescoço (88\%) e os demais estavam localizados em intestino, baço, costelas, membros superiores, peito e axila, sendo a primeira década de vida a faixa etária mais acometida. $O$ aumento de volume local foi o sinal mais observado. Após a aplicação do teste qui-quadrado a 5\% de significância, notou-se haver relação entre o gênero dos pacientes e a localização dos linfangiomas ( $p=0,0003)$. Conclusão: verificou-se que os homens, na primeira década de vida, foram os mais acometidos. Houve relação entre sexo e localização, sendo mais comuns os linfangiomas de crânio em pacientes do sexo masculino. Além disso, nos linfangiomas estudados foi observada a presença de infecção local e o tratamento foi realizado por meio de cirurgia.

Palavras-chave: Linfangioma. Hamartomas. Tumores dos vasos linfáticos. Higroma cístico.

\section{Introdução}

Os linfangiomas são tumores hamartomatosos benignos dos vasos linfáticos. Seu desenvolvimento resulta do sequestro de porções de origem linfática embriogênica primitiva que nunca se anastomosam eficientemente com canais linfáticos maiores ${ }^{1}$. O mais provável é que sejam má formações do desenvolvimento e não neoplasmas verdadeiros. Podem ser chamados de linfangiomas, higroma cístico, linfangioma circunscrito ou linfangiomatose ${ }^{2}$.

No interior da lesão, encontra-se líquido linfático rico em proteínas ${ }^{3}$. O endotélio delimitante fino é típico ${ }^{2}$, apresentando vasos linfáticos dilatados de diferentes tamanhos, revestidos por endotélio achatado sem a presença de cápsula ${ }^{1,3}$.

Os linfangiomas têm acentuada predileção por cabeça e pescoço (cerca de 50 a $70 \%$ de todos os ca$\operatorname{sos}^{2}$ ), seguidos pelas extremidades, tronco e abdômen $^{1}$, embora possam aparecer em qualquer local do sistema linfático em desenvolvimento ${ }^{4}$, sendo sua origem embriológica controversa ${ }^{5}$.

Aproximadamente, metade de todas as lesões é observada ao nascimento e $90 \%$ se desenvolvem por volta dos dois anos de idade ${ }^{2}$. Cerca de $4 \%$ de todos os tumores vasculares e $25 \%$ dos tumores benignos que afetam crianças são linfangiomas ${ }^{6}$. A ocorrência em adultos é pouco frequente e não possuem predileção por raça ou sexo ${ }^{4,7}$.

Especialista em Prótese Dentária, Mestre e Doutor em Estomatologia, professor do curso de Odontologia da Universidade de Passo Fundo.

${ }^{* * *}$ Especialista em Cirurgia Bucomaxilofacial, Mestre em Clínica Odontológica, professora do curso de Odontologia da Universidade de Passo Fundo.

*** Especialista em Patologia Bucal, Doutora em Estomatologia Clínica, professora do curso de Odontologia da Universidade de Passo Fundo. 
Apresentam-se clinicamente como tumefações nodulares e indolores, geralmente de localização superficial. Linfangiomas de língua apresentam-se com superfície pedregosa e irregular, semelhante a um grupo de vesículas translucentes ou a uma massa submucosa, nos casos em que são mais profundos. Seu aspecto superficial é comparado a "ovos de rã" ou "aglomerado de vesículas translúcidas". Devido ao denso tecido conjuntivo e à presença de músculos esqueléticos, essas lesões, em boca, não têm grande expansão $0^{9}$. Linfangiomas cervicais são mais comumente encontrados no triângulo posterior, como massas amolecidas flutuantes. No triângulo anterior, ocorrem menos comumente, porém, nessa localização podem resultar em dificuldades respiratórias ou disfagia, quando atingem grandes tamanhos ${ }^{2}$. Descritos como lesões normalmente assintomáticas, tornam-se evidentes como massas císticas, indolores, aderidas aos planos profundos ou flutuantes, recobertas por pele normal e de crescimento lento ${ }^{10}$. Os higromas císticos de grandes proporções podem envolver toda a região cervical, a face e outras estruturas, incluindo o mediastino, podendo estar associados à hidropsia e linfangiomatose difusa ${ }^{11}$. A deformidade estética é o principal sinal dessa anomalia ${ }^{2}$.

Apesar dos linfangiomas terem tendência a invadir outros tecidos, eles não têm potencial de malignização ${ }^{4}$. Quando isolados, o prognóstico é bom, porém, quando detectados intra-uterinamente, torna-se importante a realização do cariótipo fetal, por poderem estar associados a síndromes, o que torna o prognóstico mais reservado ${ }^{12}$.

Em relação ao diagnóstico diferencial, o carcinoma de células basais e o tumor de glândulas salivares com degeneração cística são incluídos. Na biópsia aspirativa essas lesões podem ser diferenciadas, pois têm características celulares neoplásicas ${ }^{13}$. Destacam-se ainda o cisto tireoglosso, rânula mergulhante e as linfadenopatias como diagnósticos diferenciais de linfangiomas da região cervical. Podem se assemelhar também, quando em menor tamanho, com mucocele e, quando possuem significativos capilares em seu interior, com o hemangioma ${ }^{14}$.

O tratamento depende do tipo, do tamanho, do envolvimento de estruturas anatômicas e de infiltração para os tecidos circundantes ${ }^{15}$. Existe o tratamento cirúrgico e os não cirúrgicos, como: radioterapia, crioterapia, cauterização, escleroterapia, administração de corticóides e de interferon, aplicação de laser de dióxido de carbono, radiofrequência, uso de OK-432 Picibanil ou solução salina hipertônica ${ }^{6,10,16-18}$. Usualmente, a excisão cirúrgica é realizada, embora a remoção total da lesão não seja possível em alguns casos pelo tamanho ou relação com outros órgãos do corpo ${ }^{2}$. As complicações da excisão incluem: dano às estruturas adjacentes, formação de fístulas, infecção e deiscência de sutura, apresentando mortalidade entre $2 \%$ e $6 \% 19$. A aspiração das lesões pode prover infecções e só é efetiva temporariamente ${ }^{20}$. A radiação não tem significativo valor terapêutico ${ }^{21}$. Ao contrário dos hemangiomas, linfangiomas não respondem bem ao tratamento esclerosante. Entretanto, alguns linfangiomas respondem bem ao tratamento com OK-432, que é um agente terapêutico esclerosante ${ }^{2}$. Soluções salinas hipotônicas que provocam inflamação do endotélio vascular podem levar à regressão total ou parcial do linfangioma, porém, em caso de cirurgia posterior, podem dificultar a realização dessa ${ }^{19}$. Há relatos de regressão espontânea, porém a sua ocorrência não é comum. A aplicação de laser de dióxido de carbono $\left(\mathrm{CO}_{2}\right)$ tem como vantagem causar pouca ou nenhuma ferida e manter o campo cirúrgico seco. Porém, sua grande desvantagem é o custo elevado do equipamento, o qual só pode ser operado por profissional capacitado ${ }^{22}$.

Tendo em vista o referencial teórico exposto, o presente trabalho objetivou realizar um levantamento dos casos de linfangiomas registrados nos laboratórios de Patologia do ICB-UPF e do HSVP entre os anos de 1987 e 2012.

\section{Materiais e método}

O presente trabalho foi submetido e aprovado pelo Comitê de Ética em Pesquisa da Universidade de Passo Fundo/RS/Brazil. Efetuou-se um levantamento epidemiológico dos casos de linfangioma registrados nos arquivos do laboratório de patologia bucal do ICB da UPF e no serviço de patologia do HSVP, entre os anos de 1987 e 2012 . Foram obtidos os dados relativos a sexo, raça e idade dos pacientes acometidos, bem como relativos à localização, ao aspecto clínico, à sintomatologia, ao tratamento efetuado e à ocorrência de recidiva das lesões.

Os dados coletados foram tabulados em planilha eletrônica e posteriormente analisados por meio de estatística descritiva de frequência e teste de Qui-quadrado a $5 \%$ de significância.

\section{Resulltados}

Foram encontrados 78 casos de linfangioma, dos quais 69 envolviam cabeça e pescoço (88\% dos casos), e os demais localizados em intestino, baço, arcos costais, membros superiores, escápula, mama, axila e coxa (Figura 1). 


\section{Localização}

" Crânio

Demais localizações

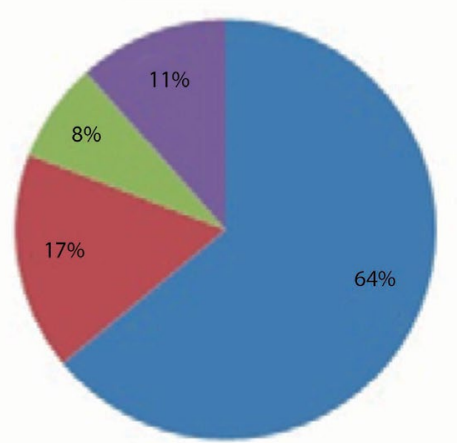

Figura 1 - Dados relativos à localização das lesões pesquisadas

Do total de casos, 53 ocorreram no sexo masculino $(68 \%)$ e 25 no feminino (32\%). Nos casos de cabeça e pescoço, encontrou-se 51 casos no sexo masculino $(74 \%)$ e 18 no feminino (26\%).

A faixa etária dos pacientes variou entre um mês e 90 anos, e a média de idade foi de 45 anos, e a faixa mais acometida, a primeira década de vida, com 20 casos (26\%), dos quais 15 ocorreram em crianças de até três anos de idade. Em seguida, constatou-se a apresentação de 12 casos na terceira década, com 12 casos (15\%) (Figura 2).

\section{Faixa Etária}
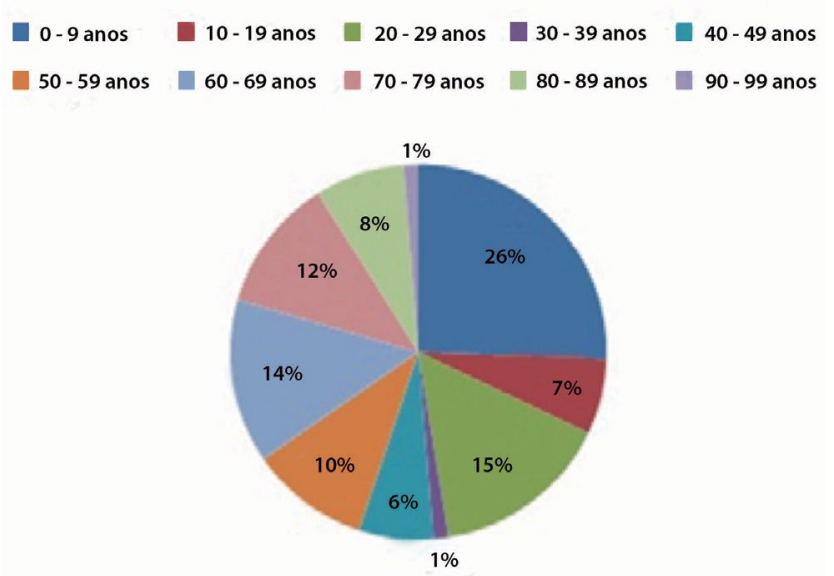

Figura 2 - Ocorrência de linfangiomas segundo a faixa etária dos pacientes

Quanto aos sinais e aos sintomas, 31\% dos casos apresentaram aumento de volume local, seguidos por $20 \%$ de casos com ausência de sinais e sintomas e apenas $4 \%$ dos casos apresentaram dor (Figura 3).

\section{Sinais e Sintomas}

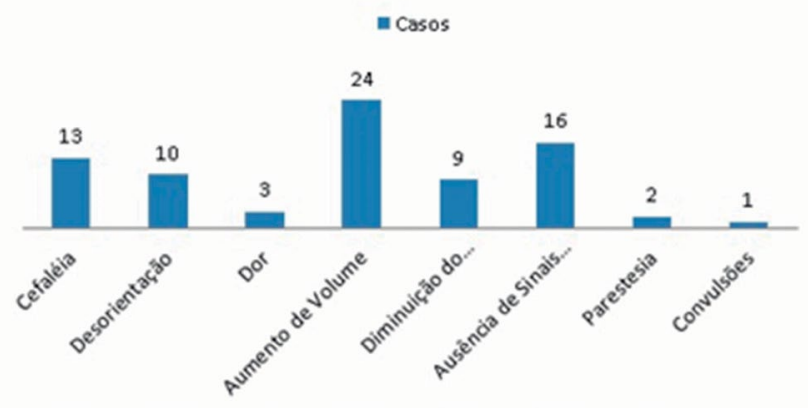

Figura 3 - Sintomatologia das lesões pesquisadas

O tratamento mais comumente realizado foi 0 cirúrgico (74\%), seguido pelo acompanhamento clínico $(18 \%)$, clínico-cirúrgico $(7 \%)$ e drenagem $(1 \%)$. A recidiva foi relatada em $8 \%$ dos casos.

Quanto à localização dos linfangiomas de cabeça e de pescoço, foram encontrados 50 casos em crânio (72\%), 13 em região cervical (19\%) e seis casos na cavidade bucal $(9 \%)$.

Considerando o gênero e a localização das lesões, os linfangiomas localizados na cabeça tiveram predileção por homens (84\%), o que não foi notado nos casos cervicais e de cavidade bucal, em que, respectivamente, $53 \%$ e $33 \%$ dos casos ocorreram em indivíduos do sexo masculino (Figura 4).

\section{Localização x Gênero}

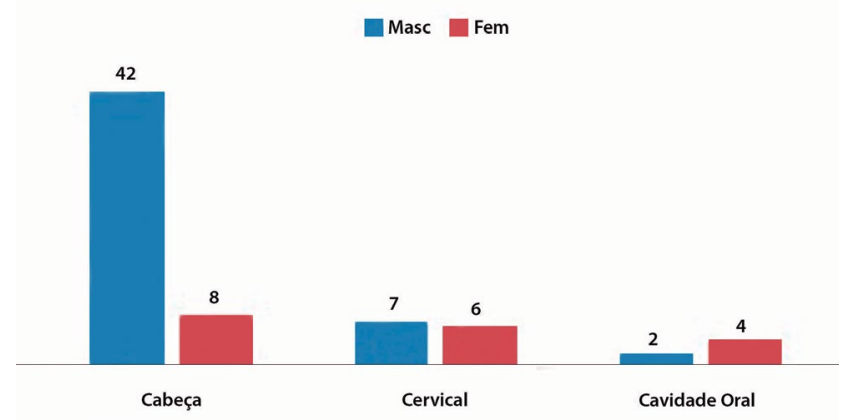

Figura 4 - Fequência dos linfangiomas, relacionando a localização dos mesmos e o gênero dos pacientes acometidos

Comparando-se as faixas etárias com as localizações, os linfangiomas de cabeça ocorrem principamente em indivíduos na sétima década de vida (28\%), em geral, associados a traumatismos no local. Já os linfangiomas cervicais e os da cavidade bucal tiveram predileção por crianças com idade entre zero e nove anos (70\% e 50\% dos casos, respectivamente) (Figura 5). 


\section{Faixa Etária x Localização}

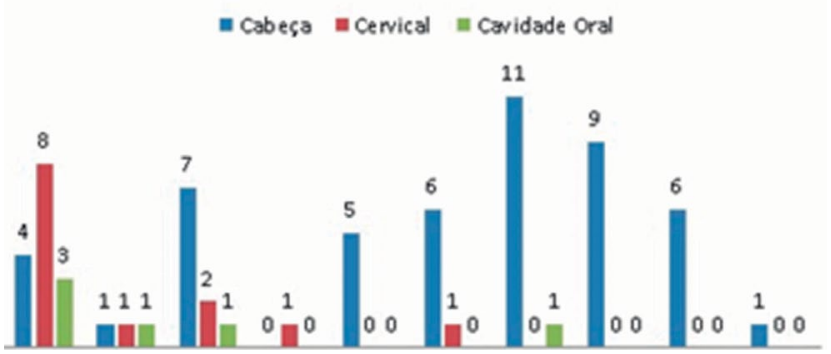

Figura 5 - Frequência dos linfangiomas relacionando localização e a faixa etária dos pacientes acometidos

Foi aplicado o teste Qui Quadrado a 5\% de significância e obteve-se $p=0,0003$ para os linfangiomas localizados no crânio em pacientes do sexo masculino. Não houve significância nas demais variáveis analisadas.

\section{Discussão}

Quanto ao aspecto clínico das lesões observadas no presente estudo, nossos achados coincidem com os da literatura ${ }^{2,14}$, os quais reportam que os linfangiomas apresentam-se como tumefações indolores e nodulares comumente localizados superficialmente, mostrando uma superfície pedregosa que se assemelha a um grupo de vesículas translucentes ou como uma massa submucosa, quando localizados mais profundamente. A cor é de uma tonalidade mais clara (leitosa-transluscente) que o tecido circunjacente.

Dos 78 casos de linfangioma encontrados no presente estudo, 69 são de localização em cabeça e pescoço (88\%), havendo relatos da preferência significativa da lesão nessas localizações (50\% a 70\%) ${ }^{2}$. Em seguida, a predileção foi pelas extremidades, depois tronco e, por último o abdômen ${ }^{1}$. Vindo ao encontro dos resultados do presente levantamento, Wiggs e Sismanis ${ }^{23}$ (1994) afirmam que o pescoço é o sítio mais comumente envolvido, sendo o terço posterior acometido com maior frequência que o anterior. Quando ocorrem nesse local são denominados higroma cístico, higroma cervical e linfangioma cavernoso, os quais causam tumefação difusa nos tecidos moles, podendo ameaçar a vida do paciente por envolver estruturas vitais no pescoço ${ }^{14}$.

Dos casos de linfangioma, $26 \%$ foram relatados na primeira década de vida, sendo $75 \%$ desses nos primeiros três anos de vida. Observa-se que $90 \%$ das lesões em crianças se manifestam em torno dos dois anos de idade ${ }^{2}$, corroborando a ideia de que a maior parte das lesões se manifesta precocemente. Porém, metade de todas as lesões é observada ao nascimento ${ }^{2}$, discordando dos achados do presente estudo, quando apenas cerca de $20 \%$ dessas foram relatadas nos primeiros anos de vida.

Neste trabalho, foi encontrada acentuada predileção da lesão pelo sexo masculino (78\% dos casos), discordando dos estudos que relatam não haver predileção por sexo ou raça ${ }^{4,7}$. Autores ${ }^{4}$ relatam também que os linfangiomas podem ser encontrados em todas as faixas etárias e com inúmeros fatores etiológicos, como traumatismos acidentais ou repetitivos, hemorragias, infecções, alterações hormonais, entre outros, concordando com os achados desta pesquisa, na qual identificou-se que grande parte dos linfangiomas em idades mais avançadas surgiram após traumatismos locais, ocasionados por quedas ou acidentes de trânsito. Encontra-se a assertiva de que a ocorrência em adultos seria pouco frequente ${ }^{8}$, discordando do achado no presente estudo, que apresentou cerca de $60 \%$ de todos os casos em adultos a partir dos 20 até os 90 anos de idade.

Em 2001, Souza e Tone ${ }^{10}$ descreveram os linfangiomas como lesões assintomáticas e indolores que se tornam evidentes como massas císticas, fato constatado em metade dos casos do presente estudo, que se apresentaram como assintomáticos (20\%) e com aumento de volume (31\%). Neville et al. ${ }^{2}$ (2009) citam ainda que os linfangiomas podem apresentar dor, sintoma encontrado em $4 \%$ dos casos estudados. Os mesmos autores ainda afirmam que os linfangiomas podem determinar prejuízos devido ao aumento de volume e à compressão dos tecidos circunjacentes, o que foi notado em alguns pacientes do presente estudo, que relataram cefaléia, desorientação, parestesia e convulsões.

Quanto ao tratamento dos linfangiomas estudados, o cirúrgico apresentou predileção (74\%). Embora autores ${ }^{2}$ citem a ressecção parcial como a mais utilizada, apenas $7 \%$ dos casos tiveram este tratamento na presente pesquisa (clínico-cirúrgico). O acompanhamento clínico foi feito em $18 \%$ dos casos, mesmo a regressão espontânea não sendo comum ${ }^{22}$.

Em 8\% dos casos tratados cirurgicamente na presente pesquisa, foi observada recidiva, sendo compatível com achados que relatam cerca de até $27 \%$ de recidivas para os linfangiomas ${ }^{19}$ e outros que citam que de $10 \%$ a $38 \%$ das lesões removidas reaparecem ${ }^{20}$.

Para Brennan et al. ${ }^{24}$ (1997), embora os hemangiomas regridam ou desapareçam com o uso de injeção de agentes esclerosantes, muitos cirurgiões atestam que os linfangiomas não respondem a essa terapêutica. A razão disso não está esclarecida, porém, é possível que diferenças na camada endotelial ou nos componentes da parede vascular possam ser contributivas. Neste estudo não foram relatados casos de tratamento de lesões por meio da esclerose.

Quando comparadas, as faixas etárias com as localizações anatômicas, linfangiomas localizados no crânio tiveram predileção pela faixa etária dos 60 aos 69 anos (28\% dos casos), em decorrência de traumas locais; já os cervicais e de cavidade oral tiveram predileção por crianças de zero até nove anos, sendo $70 \%$ e $50 \%$ dos casos apresentados nesses locais, respectivamente. 
Considerando o gênero e as localizações, apenas nos casos em crânio, os linfangiomas apresentaram predileção pelo sexo masculino, apresentando 42 casos em homens (84\%) e 8 em mulheres (16\%). Houve certo equilíbrio nas lesões cervicais, onde sete casos acometeram os homens (53\%) e seis casos as mulheres (47\%). Nas lesões de cavidade oral, dois casos relatados em sexo masculino e quatro em feminino, sem apresentar significância estatística. Significância foi observada apenas nos casos de crânio quando comparados aos gêneros, nos quais obteve-se $p=0,0003$ ao ser aplicado o teste Qui Quadrado a $5 \%$ de significância.

Não foram encontradas até o momento na literatura disponível acerca dos linfangiomas, observações que dizem respeito às relações idade $\mathrm{x}$ localizações anatômicas ou gênero $\mathrm{x}$ localizações anatômicas.

\section{Conclusões}

Com o presente estudo, após a análise estatística descritiva, constatou-se que o sexo masculino, a primeira década de vida, a localização em crânio, a presença de tumefação local e o tratamento cirúrgico foram os achados mais observados nos casos de linfangioma estudados. Após a aplicação do teste Qui-Quadrado a 5\% de significância, notou-se haver relação entre gênero dos pacientes e localização dos linfangiomas $(p=0,0003)$, sendo mais comuns os linfangiomas de crânio em pacientes do sexo masculino. Além disso, por ser uma enfermidade que acomete consideravelmente a região de cabeça e pescoço, o linfangioma constitui tema de grande interesse ao cirurgião-dentista.

\section{Abstract}

Introduction: Lymphangiomas are benign lymph vessels tumors that affect, in general, head and neck. May be congenital or injuries that arise during life. Children are the most affected. Can be surgically removed, just responding to sclerosing treatments or radiation. Its recurrence is described in about 10 to $40 \%$ of cases. Objective and Employee Methods: The aim of this study was to make a survey of the cases of lymphangioma recorded in the Pathology Laboratories of the Institute of Biological Sciences, University of Passo Fundo (ICB/ UPF) and the Hospital of Saint Vincent Paul Passo Fundo/RS (HSVP) since the year 1987 until 2012. Results: In all, 78 cases of lymphangioma were found. Of these, 69 involved the head and neck (88\% of cases) and others afflict intestine, spleen, ribs, upper limb, shoulder blade, breast, armpit and thigh and the first decade of life was the most affected age group. The local volume increase was the most observed signal. After application of chi-square test at 5\% of significance, we noted a relationship between patient gender and location of the lymphangiomas ( $p=0.0003$ ). Conclusions: It was noted that men in the first decade of life were the most affected. There was a relationship between gender and location, being lymphangiomas more common in skull of males. Furthermore, in lymphangiomas studied we noted the presence of local infection and the treatment was by surgery.

Keywords: lymphangioma, hamartomas, neoplasms of lymphatic vessels, cystic hygroma.

\section{Referências}

1. Balakrishnan, A, Bailey, CM. Lymphangioma of the tongue, A review of pathogenesis, treatment and the use of surface laser photocoagulation. J Laryngol Otol 1991; 105(11):924-9.

2. Neville BW, Allen CM, Damm DD, Bouquot JE. Patologia Oral e Maxilofacial. 3ra edición. Rio de Janeiro: Guanabara Koogan; 2009.

3. Hellman JR, Myer CM, Prenger EC. Therapeutic alternatives in the treatment of life-threatening vasoformative tumors. Am J Otolaryngol 1992; 13(1):48-53.

4. Woodward PJ, Sohaey R, Kennedy A, Koeller KK. A comprehensive review of fetal tumors with pathologic correlation. Radiographics 2005; 25:215-42.

5. Kennedy TL. Cystic hygroma-lymphangioma: a rare and still unclear entity. Laryngoscope 1989; 99:1-10.

6. Fernandez GMD. Limphangioma. Available at: <http:// emedicine.com/derm/topic866.htm>. Acesso em mar. 2012.

7. Koeller KK, Alamo L, Adair CF, Smirniotopoulos JG. Congenital cystic masses of the neck: radiologic-pathologic correlation. Radiographics 1999; 19:121-46.

8. Ganesh C, Sangeetha GS, Narayanan V, Umamaheswari TN. Lymphangioma circumscriptum in an adult: an unusual oral presentation. J Clin Imaging Sci 2013; 29(3):44. doi:10.4103/2156-7514.120779. eCollection 2013.

9. Mosca RC, Pereira GA, Mantesso A. Cystic hygroma: characterization by computadorized tomography. Oral Surg Oral Med Oral Pathol Oral Radiol Endod 2008; 105(5):65-9.

10. Souza RJSP, Tone LG. Tratamento clínico do linfangioma com alfa-2a-interferon. J Pediatria 2001; 77(2):139-42.

11. Davies D, Rogers M. Morphology of limphatic malformations: a pictorial review. Australas J Dematol 2009; 41:1-5.

12. Kaminopetros P, Jauniaux E, Kane P, Weston M, Nicolaides $\mathrm{KH}$, Campbell DJ. Prenatal diagnosis of na extensive fetal lymphangioma using ultrasonography, magnetic resonance imaging and cytology. Br J Radiol 1997; 70:750-3.

13. Mandel L. Parotid area lymphangioma in adult: case report. J Oral Maxilofac Surg 2004; 62(10):1320-3.

14. Regezi JA, Sciubba JJ. Patologia Oral: correlações clinicopatológicas. $5^{\text {a }}$ edición. São Paulo: Elsevier; 2008.

15. Ethunandan M, Mellor TK. Hemangiomas e malformações vasculares da região maxilofacial. Br J Oral Maxilofac Surg 2006; 44:263-72.

16. Lapidoth M, Ackerman L, Amitai DB, Raveh E, Kalish E, David M. Treatment of lymphangioma circumscriptum whit combined radiofrecuency current and $900 \mathrm{~nm}$ diode laser. Dermatol Surg 2006; 32(6):790-4.

17. Bozkaya S, Ugar D, Karaca I, Ceylan A, Uslu S, Baris E, Tokman B. The treatment of lymphangioma in the bucal mucosa by radiofrequency ablation: a case report. Oral Surg Oral Med Oral Pathol Oral Radiol Endod 2006; 102(5):28-31. 
18. Okubo T, Shimada T, Narita Y. A success ful case report on intralesional OK-432 therapy for cystic mediastinal lymphangiomas. Kyobu Geka 1998; 51(12):1017-21.

19. Vasconcelos BN, Benez MDV, Bressan AL, Oliveira EF. Higroma cístico de face com involução após infecção local. An Bras Dermatol 2011; 86(1):135-7.

20. Akbay E, Cevik C, Arli C. Perioperative difficulties and early postoperative complications of transoral approach in mouth base surgery. J Craniofac Surg 2014; 25(2): e 143-8

21. Goshen S, Ophir D. Cystic hygroma of the parotid gland. J Laryngol Otol 1993; 107:855-7.

22. Gama SKC, Araújo TM, Pinheiro ALB. Benefits of the use of the $\mathrm{CO}_{2}$ laser in orthodontics. Lasers Med Sci 2008; 23:45965.

23. Wiggs WJ, Sismanis A. Cystic higroma in the adult: two case reports. Otolaryngol Head Neck Surg. 1994; 110(2):239-41.

24. Brennan TD, Miller AS, Chen S. Lymphangiomas of the oral cavity: a clinicopathologic, immunohistochemical, and electron-microscopic study. J Oral Maxillofac Surg 1997; 55(9):932-5.

Endereço para correspondência:

Soluete Oliveira da Silva

Rua Padre Nóbrega, 475/301

Bairro Lucas Araújo Passo Fundo-RS

Fone: 54 3316-8402

E-mail: soluete@upf.br

Recebido: 05/05/2014. Aceito: 07/08/2014. 